\title{
Criminologie
}

\section{Les liaisons dangereuses : Foucault et la criminologie}

\section{Dany Lacombe}

Volume 26, numéro 1, 1993

Michel Foucault et la (post) modernité

URI : https://id.erudit.org/iderudit/017330ar

DOI : https://doi.org/10.7202/017330ar

Aller au sommaire du numéro

Éditeur(s)

Les Presses de l'Université de Montréal

ISSN

0316-0041 (imprimé)

1492-1367 (numérique)

Découvrir la revue

Citer cet article

Lacombe, D. (1993). Les liaisons dangereuses : Foucault et la criminologie.

Criminologie, 26(1), 51-72. https://doi.org/10.7202/017330ar

\section{Résumé de l'article}

With Discipline and Punish, Michel Foucault offered the social sciences a theory of power, and conceptual tools that radically transformed law reform studies. In criminology, for example, the social constructionist tradition, by drawing on Foucault's notion of power, increasingly inscribed law reform studies in a narrative of the dispersal of social control. Attempts to reform the criminal justice system are understood in terms of the increased penetration and expansion of social control into the whole of the social body ; thus, "nothing works !" In this article, I intend to challenge this conventional wisdom on law reform and the dispersion of social control, by demonstrating that it is founded on an essential-ist notion of power that we cannot attribute to Foucault. In light of his work on sexuality, and governmentality, I will examine how Foucault's productive notion of power is better understood in terms of "mechanisms for life", strategies that both constrain — through objectifying techniques — and enable - through subjectifying techniques — agency. The implications of Foucault's productive notion of power for law reform are examined in terms of methodological considerations.
Ce document est protégé par la loi sur le droit d'auteur. L'utilisation des services d’Érudit (y compris la reproduction) est assujettie à sa politique d'utilisation que vous pouvez consulter en ligne.

https://apropos.erudit.org/fr/usagers/politique-dutilisation/ 
With Discipline and Punish, Michel Foucault offered the social sciences a theory of power, and conceptual tools that radically transformed law reform studies. In criminology, for example, the social constructionist tradition, by drawing on Foucault's notion of power, increasingly inscribed law reform studies in a narrative of the dispersal of social control. Attempts to reform the criminal justice system are understood in terms of the increased penetration and expansion of social control into the whole of the social body; thus, "nothing works!" In this article, I intend to challenge this conventional wisdom on law reform and the dispersion of social control, by demonstrating that it is founded on an essentialist notion of power that we cannot attribute to Foucault. In light of his work on sexuality, and governmentality, I will examine how Foucault's productive notion of power is better understood in terms of "mechanisms for life", strategies that both constrain through objectifying techniques - and enable - through subjectifying techniques - agency. The implications of Foucault's productive notion of power for law reform are examined in terms of methodological considerations.

L'importance de Michel Foucault en criminologie, depuis les quinze dernières années, est remarquable. Comme le souligne Stanley Cohen, traiter de la criminologie aujourd'hui sans évoquer Foucault, c'est comme parler de l'inconscient sans mentionner Freud (1985, p. 10). Avec la publication de Surveiller et punir: la naissance de la prison (1975), Foucault a donné à la criminologie un nouveau mode de pensée et de nouveaux concepts qui, selon David Garland $(1986$, p. $866 ; 1990)$, ont radicalement changé le discours dans lequel la réforme pénale était typiquement pensée par la criminologie. Ces concepts - «pouvoir-savoir», «société

1. L'auteure est titulaire d'une bourse de recherche postdoctorale du Conseil de recherches en sciences humaines du Canada. Elle est attachée au Centre de Recherches Sociologiques sur le Droit et les Institutions Pénales (CESDIP), 4, rue de Mondovi, 75005 Paris, de même qu'au Centre of Criminology, University of Toronto, 130 St. George St., Toronto, Ontario, M5S IA1.

Elle remercie Bessie Leconte du GERN (Paris) et Claude Faugeron du CESDIP-CNRS (Paris) pour leur lecture attentive, ainsi que le Conseil canadien de la recherche en sciences humaines pour son apport financier. 
disciplinaire», «micro-pouvoirs» - ont permis aux analystes de déconstruire à la fois la conception libérale des réformes pénales, en particulier de la prison, comme une avancée humaniste par rapport au système de punition barbare et cruelle d'une société traditionnelle, et la conception marxiste qui comprend la prison comme un épiphénomène du mode de production. Pour Foucault, l'histoire des réformes pénales est celle de la dispersion d'un nouveau mode de domination, qui s'exerce par le pouvoir disciplinaire, lequel, à travers des techniques d'objectivation, de classification et de normalisation des individus, se déploie dans tout le corps social.

Les concepts de Foucault ont également permis d'étendre le champ de la criminologie à l'ensemble des institutions sociales, en particulier à celles produites par l'expansion de l'État providence dans les années 1960. Ainsi, autant les institutions qui nous contrôlent de façon répressive (la prison, l'asile, les tribunaux, etc.) que celles qui règlent nos vies quotidiennement (l'éducation, la santé, la sécurité sociale, etc.), ont été analysées pour leurs effets disciplinaires et normalisants, c'est-à-dire pour leurs effets de contrôle, de domination, et d'objectivation. Ces études s'appuyant sur la conception foucaldienne de la pénalité en tant que technique productive d'un pouvoir-savoir ont contribué, cependant, a la prolifération d'un savoir fonctionnaliste et instrumentaliste du pouvoir et de la réforme du droit ${ }^{2}$. Ainsi, à l'instar de Foucault, nous voyons se développer en criminologie une vaste documentation démontrant de façon ingénieuse et cynique que toute tentative d'amélioration du système, en particulier du système pénal, ne sert qu'à la dispersion et à l'expansion du contrôle dans le corps social. Par conséquent, la réforme apparaît à la fois comme le support et le produit d'une structure dominante dans notre société moderne, le contrôle social ${ }^{3}$.

Dans cet article, j'aimerais révaluer ce savoir sur la réforme du droit, qui est devenu conventionnel en criminologie, et qui s'appuie sur une notion du pouvoir que l'on attribue à Foucault. En fait, je veux poser la question suivante: si nous acceptons que les réformes pénales aient échoué

2. David Garland souligne ce phénomène pour les études portant sur la prison: "Foucault's emphatic depiction of punishment as a technology of power-knowledge and his primarily political account of its historical development have produced an instrumental and functionalist conception of punishment in which penal practice is always shaped exclusively by the requirements of social control and in which its design is always calculated to maximize control effects." (Garland, 1990, p. 163).

3. Voir entre autres les travaux de Cohen, 1979, 1983, 1985; Ericson, 1985, 1987; Ericson et Baranek, 1982, 1985; Chan et Ericson, 1981; Giffen et Lambert, 1988; Ben-Yehuda, 1985, 1986; Small, 1988; Watney, 1987. 
quant au grand projet de la modernité comme un «fait empirique ${ }^{4} »$, comment se fait-il que nous concevions ce constat d'échec en terme de pénétration toujours plus oppressive et diffuse d'une structure restrictive de domination et de contrôle? Cette conception est-elle due au nouveau mode de pensée et aux concepts proposés par Foucault (qui seraient essentialistes, instrumentalistes et fonctionnalistes), ou à la façon dont ceux-ci, en particulier sa notion du pouvoir, ont été appropriés par les analystes de la réforme? David Garland semble être d'avis que les concepts de Surveiller et Punir (1975) ont contribué à la production d'une conception du pouvoir dont la fonction principale serait d'ordre répressif: la production de techniques disciplinaires y est pensée en termes de domination, de normalisation et de contrôle. Il affirme :

[T] he story which Discipline and Punish tells - and the one which most influences current work in the sociology of punishment - is one of meticulous domination and thoroughgoing control, so that we are left with the distinct impression that society's practices of normalization its imposition of standards upon conduct - are oppressive in all their aspects. Despite Foucault's later stress upon «subjectification ", Discipline and Punish tells a story of objectification of human beings through the use of power-knowledge, and its critique of power and society is largely an extension of this imagery of dehumanizing domination. (Garland, 1990, pp. 169-170).

Bien qu'étant en partie d'accord avec la critique de Garland, je pense qu'il est grand temps que nous nous intéressions, en criminologie, à l'auvre de Foucault plutôt qu'à un seul de ses textes, qui semble d'ailleurs n'avoir eu d'importance pour les criminologues que par le fait qu'il traite explicitement d'un thème central en criminologie, la prison. J'essaierai de démontrer qu'à la lumière des écrits de Foucault nous pouvons béneficier pleinement de l'importance de la conception du pouvoir qu'il a esquissée dans Surveiller et punir: un mécanisme de production à la fois de relations d'objectivation (dans le sens d'assujettissement par les normes) et de relations de «subjectivation» (dans le sens de résistance aux normes). Ce double mécanisme du pouvoir, avec ses effets à la fois totalisants (ou intégrants) et individualisants (ou excluants), élude la possibilité de penser

4. Sur la question de ce «fait» empirique, qui semble reposer clairement sur des données statistiques sans équivoque, voir l'excellente analyse de McMahon (1992). Elle démontre, dans le cas de l'Ontario, que l'augmentation du nombre de personnes ayant à faire avec le systène correctionnel à la suite de l'implantation d'altematives à la prison n'est pas fondée statistiquement. 
la réforme en fonction d'un essentiel déploiement du contrôle dans tout le corps social. En fait, comme je tenterai de le démontrer, la conception du pouvoir en tant que mécanisme de production, chez Foucault, est un apport considérable à notre compréhension de la relation entre la structure sociale et l'agent social.

\section{LES RÉFORMES PÉNALES : CONSTAT D'ÉCHEC ET DE DISPERSION DU CONTRÔLE SOCIAL}

Le constat de l'échec de la prison comme solution éclairée, rationnelle et juste du système de punition barbare qui la précédait a grandement inspiré les analyses des réformes pénales depuis les années 1960 pour améliorer le système de justice criminelle. Ces réformes qui se fondaient, comme la prison auparavant, sur le récit de la marche vers le progrès, ont été examinées avec le même regard désenchanté que l'a été le récit sur la prison: les alternatives à la prison ne semblent ni améliorer ni humaniser le sort des gens qui entrent en contact avec le système de justice criminelle. À la suite de l'échec et de la prison et des alternatives à la prison pour enrayer les pratiques oppressives du système de punition des sociétés occidentales contemporaines, il s'est formé un consensus académique sur la réforme pénale. Ce consensus correspond au savoir selon lequel les réformes pénales ne modifient pas fondamentalement les pratiques oppressives du système de justice criminelle, mais ne font que les reproduire dans des formes différentes, d'où, en criminologie, la fameuse expression: nothing works!

Stanley Cohen exprime ainsi ce constat d'échec des politiques pour transformer le système de justice criminelle des sociétés contemporaines :

the most fundamental fact about what is going on in the new agencies is that it is much the same as what went on and is still going on in the old system (1985, p. 79).

En fait, "what is going on», c'est-à-dire l'effet de la réforme, correspond à quelque chose de plus qu'à un échec. Cohen affirme qu'un changement s'est définitivement produit avec l'instauration d'alternatives au système carcéral. S'appuyant sur la thèse du raffinement et de l'obscurcissement des mécanismes de contrôle et de disciplinarité caractéristiques de la société moderne, identifiés par Foucault (1975), Cohen affirme que le changement apporté par les politiques pénales pour transformer la prison est le suivant: 
a gradual expansion and intensification of the system; a dispersal of its mechanisms from more closed to more open sites and a consequent increase in the invisibility of social control and the degree of its penetration into the social body (1985, pp. 83-84).

Comme le dit Foucault, la réforme pénale apparâ̂t comme une «technologie du pouvoir», un mécanisme producteur d'un type de contrôle qui devient de plus en plus difficile à cerner; si bien que l'horizon sur lequel se distinguent les formes de contrôle formel et informel s'obscurcit progressivement. La réforme produit ainsi une pratique paradoxale: à la fois the thinning of the mesh the widening of the net du contrôle social (Cohen, 1983,1985 ).

La thèse de la dispersion du contrôle dans tout le corps social - thèse encapsulée dans l'expression foucaldienne favorite des criminologues, la « société disciplinaire» - a contribué à élargir le champ de la criminologie à tout le corps «carcéral». La criminologie critique s'est alors mise à dévoiler tous les micro-pouvoirs et les technologies de contrôle de la «société disciplinaire». Ainsi Ericson et Baranek (1982) focalisent leur conclusion sur la subordination de l'accusé dans le système de justice criminelle canadien à la position du citoyen devant la loi: les droits de la personne humaine, la notion de justice, et la philosophie de la justice équitable (due process) sont tous réduits à des mécanismes de contrôle social qui le renforcent (voir également, McBarnett, 1981). Ericson (1985, 1987) affirme que la réforme du droit, en général, est une simple rhétorique (reform talk) dont le but principal est de reproduire la structure de domination caractérisant les sociétés modernes. Ainsi la Charte canadienne des droits de la personne n'est que du social control talk qui semble avoir été promulguée pour favoriser le contrôle social et rien de plus (Ericson, 1985). Shearing et Stenning (1984) nous présentent Disney World comme la quintessence du pouvoir disciplinaire non pénal : les barrières, le monorail, le parking, les instructions, les préposés et les personnages Disney - bref tout ce qui se trouve dans cet univers «fantastique» - dissimulent derrière une façade d'amusement, de sécurité et de confort leur «vraie" nature; ce sont là des stratégies de contrôle normalisant les individus.

En somme, à l'instar de Foucault, s'est établi en criminologie un savoir fondê sur une conception du pouvoir, entendue comme une puissance qui déploie son rayonnement fatal sur tout le corps social. Dans cette vision de la réalité, toute politique et tout mouvement de réforme fondés sur la nécessité de transformer la société afin de l'améliorer, de l'humaniser, de la rendre plus équitable et juste, se révèlent être des stratégies de domination. 
Les réformes deviennent d'inéluctables technologies du pouvoir qui assurent une pénétration plus grande et étendue de ce vaste filet de plus en plus serré qui étranglerait et la société et les individus.

La logique circulaire de cette conception de la réforme et du contrôle est articulée à partir d'une vision essentialiste de la société. En fait, les études sur la réforme conçoivent la société comme une sphère totalisante, organisée par l'État ou par la classe dominante, et centrée autour d'une structure, le contrôle, qui doit se reproduire afin de survivre. La conception «productive» du pouvoir qui s'inscrit dans cette logique est essentiellement répressive : la réforme en tant que technique de pouvoir ne produit que des effets de domination. Cette conception est loin d'être celle proposée par Foucault. Bien que Surveiller et punir (1975) décrit un état de police où les disciplines constituent des pratiques de la norme, la «police" et les disciplines ne sont pas essentiellement des pratiques normatives de surveillance et du maintien de l'ordre. En fait. Foucault parle de «la police» en tant qu'ensemble de mécanismes pour assurer le «bien public»:

L'ensemble des moyens qu'il faut mettre en cuvre pour assurer, au-delà de la tranquilité et du bon ordre, le «bien public», tel est, en gros, ce qu'en Allemagne et en France on a appelé la «police». «Ensemble des lois et règlements qui concement l'intérieur d'un État. qui tendent à affermir et à augmenter sa puissance, à faire un bon emploi de ses forces et à procurer le bonheur de ses sujets" (J. Von Justi). Ainsi entendu (sic), la police étend son domaine bien plus loin que la surveillance et le maintien de l'ordre. Elle a à veiller à l'abondance de la population [...] à l'activité des individus [...] à la circulation des choses et des gens [...] La police, on le voit, c'est toute une gestion du corps social. Ce terme de «corps», il ne faut pas l'entendre de façon simplement métaphorique car il s'agit d'une matérialité complexe et multiple [...] la police, comme ensemble institutionnel et comme modalité d'intervention, a en charge l'élément physique du corps social: la matérialité, en quelque sorte, de cette société civile dont on cherchait à la même époque à penser par ailleurs le statut juridique (Foucault, in Barret-Kriegel, 1989. p. 224).

Nous verrons plus loin la centralité des mécanismes visant la maximisation de «la population» dans la conception du pouvoir, chez Foucault. Pour l'instant, j'aimerais démontrer comment, à partir de son ceuvre, on ne peut soutenir la thèse d'une pénétration sans équivoque d'une stratégie de répression et de domination. Le pouvoir, bien que parfois producteur de contrôle, ne se limite pas qu'à cette production. L'idée que le pouvoir fabrique des individus (Foucault, 1975, p. 172) me semble beaucoup plus 
centrale dans la recherche de Foucault et beaucoup plus prometteuse pour comprendre les mécanismes de normalisation et de résistance.

\section{LA CONCEPTION PRODUCTIVE ET RELATIONNELLE DU POUVOIR CHEZ FOUCAULT}

\section{a) Surveiller et punir et le pouvoir-savoir}

Le pouvoir, nous dit Foucault, doit être pensé en termes de ses effets de production. Cela implique une nouvelle approche:

Il faut cesser de toujours décrire les effets de pouvoir en termes négatifs: il «exclut», il «réprime», il «refoule», il «censure», il «abstrait», il «masque», il «cache». En fait, le pouvoir produit; il produit du réel; il produit des domaines d'objets et des rituels de vérité. L'individu et la connaissance qu'on peut en prendre relèvent de cette production (Foucault, 1975, p. 196).

Dans Surveiller et punir (1975) Foucault démontre cet aspect producteur du pouvoir en examinant entre autres le rapport entre la punition, une technique du pouvoir, et les sciences humaines. Il affirme qu'a partir des pratiques modernes de punitions (l'observation, l'examen, la mesure, la classification, la surveillance, etc.) émerge une connaissance systématique des individus permettant simultanément et le développement des sciences humaines (la psychologie, la criminologie, la sociologie, etc.) et l'exercice d'un type de pouvoir et de contrôle sur les individus ainsi fabriqués. L'analyse de Foucault révèle donc que les savoirs, en tant que formes de pensée et d'action, sont reliés de façon complexe aux opérations du pouvoir. En fait, le pouvoir et le savoir sont intimement lies par un processus de constitution mutuelle: l'un impliquant nécessairement l'autre. Foucault parle ainsi du «pouvoir-savoir»:

Il n'y a pas de relation de pouvoir sans constitution corrélative d'un champ de savoir, ni de savoir qui ne suppose et ne constitue en même temps des relations de pouvoir. $(1975$, p. 32).

Le «pouvoir-savoir» suppose qu'il n'y a pas d'affirmation qui existerait dans un champ dépourvu de pouvoir, qu'il n'y a pas de vérité sans une politique de vérité. Au lieu de chercher à déterminer le pouvoir, c'est-à-dire à le définir en tant qu'essence, le concept du «pouvoir-savoir» porte à s'interroger sur le comment du pouvoir, c'est-à-dire sur les procédures de son exercice. Ainsi, comme le souligne bien Ewald (1975), la question que 
Foucault pose aux vérités et à leurs discours est descriptive plutôt que déterminative du pouvoir: «De quelles procédures de production relevezvous? De quelles relations de pouvoir procédez-vous? Quels types d'assujettissement (ou de libération) produisez-vous?» (1975, p. 1230).

Cette méthode d'investigation des savoirs ne s'inscrit pas dans une conception unitaire du pouvoir, comme c'est le cas avec le savoir conventionnel sur la réforme du droit. Alors que Surveiller et punir (1975) démontre effectivement le déploiement d'une micro-physique du pouvoir qui s'est opérée à travers le contrôle social, la conception du pouvoir chez Foucault $n$ 'en est pas pour autant fixée dans un système unitaire de contrôle et de répression. En fait, sa vision de la sphère pénale exclut l'idée d'une structure sociale préconçue. Son mode d'analyse évite la suggestion que la société est un tout cohérent pouvant se comprendre à travers des modeles structuraux ou des concepts globaux (Garland, 1990, p. 133). Lors d'une entrevue précédant la réimpression du Panoptique de Bentham, Foucault déclare l'impossibilité de déterminer le pouvoir, de trouver son origine:

Mais si tu me demandes: cette nouvelle technologie de pouvoir a-t-elle historiquement son origine dans un individu ou dans un groupe déterminé d'individus qui auraient décidé de l'appliquer pour servir leurs intérêts et rendre leur corps social utilisable par eux, je répondrai: non. Ces tactiques ont étế inventées. organisées à partir de conditions locales et d'urgences particulières. Elles se sont dessinees, morceau par morceau, avant qu'une stratégie de classe les solidifie en vastes ensembles cohérents. Il faut noter d'ailleurs que ces ensembles ne consistent pas en une homogénéisation mais bien plutôt en un jeu complexe d'appuis que prennent les uns sur les autres. les différents mécanismes de pouvoir, qui restent bien spécifiques (Foucault, 1977a, p. 24).

Le refus de penser le pouvoir en termes de sa détermination, de son origine, ou de son essence implique, selon Deleuze (1975), que le pouvoir n'est pas une propriété localisée dans une institution (l'État), subordonnée à une structure (l'économie), dont le mode d'action serait instrumental, répressif et contraignant. À la vision structuraliste (instrumentaliste, fonctionnaliste et essentialiste) du pouvoir, Foucault en oppose une autre: il soutient que le pouvoir est une stratégie qui implique des relations de vérité entre individus et qui s'exerce à travers des techniques, des «micro-pouvoirs » constituant des individus et des savoirs et constitués par eux.

Afin de mieux apprécier la qualité non essentialiste et non unitaire de la conception du pouvoir chez Foucault, il est utile d'examiner L'Histoire de la sexualité : la volonté de savoir (1976). Sa critique de «l'hypothèse 
répressive» est d'une importance capitale pour comprendre comment la fabrication d'individus à travers le pouvoir ne s'inscrit pas dans une logique de domination. Comme on le verra, le pouvoir pour Foucault s'inscrit dans une logique de production de mécanismes à la fois d'objectivation et de subjectivation.

\section{b) L'Histoire de la sexualité : la volonté de savoir: subjectivation et résistance}

Dans L'Histoire de la sexualité (1976), Foucault s'en prend à l'hypothèse répressive; à la conception selon laquelle l'Europe serait passée d'une période de relative ouverture quant au corps et à la sexualité à une période caractérisée par une augmentation croissante de la répression et de l'hypocrisie sexuelle. Le sexe, dit-on, serait devenu utilitaire, sans plaisir, relégué à la famille dans le but principal de reproduire l'espèce. L'attrait de ce récit, selon Foucault, vient du fait que la répression sexuelle est pensée comme culminant dans l'ère victorienne et peut donc être facilement associé à la montée du capitalisme et du pouvoir de la bourgeoisie. Ainsi, le sexe aurait été réprimé car il allait à l'encontre de l'éthique du travail du capitalisme. La répression du sexe serait donc un résultat direct de l'opération du pouvoir sous le capitalisme : un pouvoir s'exprimant en tant que loi, instrument qui réprime, censure, fait taire, ou nie.

Au discours qui exige la prohibition du sexe, Foucault oppose celui de la production, au cours des trois derniers siècles, d'une multiplicité de discours sur le sexe: "la mise en place [d']un appareillage à produire sur le sexe des discours, susceptibles de fonctionner et de prendre effet dans son économie même» (Foucault, 1976, p. 33). Cette explosion de la «mise en discours » du sexe (Foucault, 1976, p. 21) s'exprime moins en termes d'une prohibition que d'un souci d'intérêt public; le sexe commence à être classifié, spécifié, catégorisé et quantifié afin d'être utile et de fonctionner pour le plus grand bien de tous. Foucault affirme que:

Le sexe, ça ne se juge pas seulement, ça s’administre. Il relève de la puissance publique; il appelle des procédures de gestion; il doit être pris en charge par des discours analytiques. Le sexe, au XVIII ${ }^{e}$ siècle, devient affaire de "police». [...] Police de sexe: c'est-à-dire non pas rigueur d'une prohibition mais nécessité de régler le sexe par des discours utiles et publics (1976, p. 35).

L'explosion discursive et la gestion du sexe prennent forme dans une variété de foyers de relations de pouvoirs, tels qu'en psychiatrie, médecine, démographie, biologie, pédagogie, politique, éthique, etc. Si bien que l'on ne peut limiter le champ d'action du pouvoir à un discours particulier; il 
correspond plutôt à une intensification et à une multiplication des discours produits par différents mécanismes operant dans des institutions différentes. Par conséquent, parler du pouvoir en termes d'une logique d'expansion du contrôle social, comme les analyses de la réforme du droit le supposent. n'a pas de sens. Foucault lui-même critique cette vision :

Cen'est donc pas simplement en termes d'extension continue qu' il faut parler de cette croissance discursive; on doit y voir plutôt une dispersion des foyers d'où se tiennent ces discours, une diversification de leurs formes et le déploiement complexe du réseau qui les relie (1976, p.47).

La résistance de Foucault à penser le pouvoir en terme d'extension continue d'un discours s'explique aussi par son refus de localiser le pouvoir dans un foyer spécifique tel que l'État. Foucault rejette l'importance analytique que les marxistes, en particulier, donnent à l'État. Il affirme que l'importance de l'État vient du fait qu'il est un concept théorique central en politique. En réalité, cependant, l'État est loin d'être l'entité déterminante que l'on a construit. Même si la critique de Foucault est dirigée expressément contre l'analyse marxiste de l'État, elle s'applique très bien aux analyses de la réforme du droit, qui reposent sur une conception unitaire du contrôle centré dans l'État.

Nous savons quelle fascination exerce aujourd'hui l'amour ou la phobie de l'État; nous savons combien on s'occupe de l'État; nous savons combien on s'occupe de la naissance de l'État et de son histoire, de ses progressions, de son pouvoir et de ses abus, etc. Cette valorisation excessive du problème de l'État, nous la trouvons essentiellement sous deux formes: une forme immédiate, affective et tragique, c'est le lyrisme du monstre froid qui se dresse devant nous; mais il y a une deuxième manière de surévaluer le problème de l'État — de manière paradoxale, car apparemment réductrice —, c'est l'analyse qui consiste à réduire l'État à un certain nombre de fonctions, comme par exemple le développement des forces productrices, la reproduction des rapports de production; ce rôle réducteur de l'État, par rapport à l'autre, le rend toujours absolument essentiel comme cible à atteindre et comme position privilégiée à occuper. Mais, l'État, aujourd'hui probablement, pas plus que dans le cours de son histoire, n'a cette unité, cette individualité, cette fonctionnalité rigoureuse et je dirais même cette importance. Après tout, l'État n'est peut-être qu'une réalité composite, une abstraction mythifiée, dont l'importance est beaucoup plus limitée que ce que l'on croit. Peut-être ce qui est important pour notre modernité, c'est-à-dire pour notre actualité, ce n'est pas tant l'étatisation de la société mais la «gouvernementalisation» de l'État (Foucault, 1986, pp. 14-15). 
J'examinerai cette idée de «gouvernementalité » plus loin. Pour le moment, je veux revenir à un des problèmes centraux de Foucault, la fabrication d'individus.

Alors que Surveiller et punir (1975) démontre la fabrication d'individus à travers un processus d'objectivation - dans le sens d'assujettissement à une norme -, L'Histoire de la sexualité (1976) examine comment l'individu se construit lui-même à partir d'un processus de subjectivation - dans le sens de résistance à une norme. Contrairement à l'hypothèse répressive qui pense la sexualité comme un moyen pour opprimer et réguler les masses, Foucault soutient que les discours du sexe à la fin du XVIII ${ }^{\mathrm{e}}$ siècle n'ont pas été utilisés initialement pour réprimer «l'autre». Au lieu d'être une stratégie de répression, le déploiement des discours sur le sexe correspond beaucoup plus à une stratégie permettant l'émergence de la bourgeoisie en tant que classe. C'est à travers les discours sur le sexe que la bourgeoisie s'est donnée un corps pour elle-même, qu'elle s'est établie comme une classe distincte à la fois de la décadence de l'aristocratie et de l'ignorance des masses. L'intensification des discours sur le sexe, donc, représente d'abord l'affirmation d'une subjectivité de classe plutôt qu'une stratégie de répression :

ce n'est point, semble-t-il, comme principe de limitation du plaisir des autres que le dispositif de sexualité a été mis en place par ce qu'il était de tradition d'appeler les «classes dirigeantes". Il apparaît plutôt qu'elles l'ont d'abord essayé sur elles-mêmes. [...] Plutôt que d'une répression sur le sexe des classes à exploiter, il fut d'abord question du corps, de la vigueur, de la longévité, de la progéniture, et de la descendance des classes qui «dominaient ». C'est là que fut établi, en première instance, le dispositif de sexualité, comme distribution nouvelle des plaisirs, des discours, des vérités et des pouvoirs. Il faut y soupçonner l'auto-affirmation d'une classe, plutôt que l'asservissement d'une autre (Foucault, 1976, pp. 162-163).

On voit bien, ici, que l'effet de ce dispositif de sexualité n'est pas simplement l'expansion du contrôle social. En fait, Foucault parle de la production de bio-pouvoirs: des mécanismes qui investissent la vie, qui la problématisent, la prennent en charge et la maximisent (Foucault, 1976, p. 162; pp. 188-191). Ainsi à travers l'organisation et l'élaboration de procédés de pouvoir-savoir, la bourgeoisie a entrepris non seulement de contrôler son propre corps mais de le transformer. Elle s'est donnée un corps pour elle-même, un corps qu'elle a dû maximiser en le protégeant, en le préservant et en le cultivant de telle sorte qu'il puisse retenir sa spécificité, son statut et sa valeur. C'est à travers cette stratégie d'invention d'un corps 
pour soi que la bourgeoisie a pu, ultérieurement, utiliser le dispositif de la sexualité comme technique d'assujettissement du prolétariat.

C'est dans la discussion des sexualités périphériques que Foucault illustre le mieux la façon dont le pouvoir, à travers des mécanismes objectivants et subjectivants, est impliqué dans la construction de l'individu. Il souligne que le souci des sexualités périphériques subit un changement majeur au XIX $X^{\mathfrak{e}}$ siecle, ce qui entraîne une nouvelle caractérisation des individus. Par exemple, selon les anciens codes civil ou canonique, la sodomie était prohibée en tant qu'acte illégal. L'accent était mis sur la nature juridique de l'acte - une infraction légale - plutôt que sur l'auteur de l'acte, qui n'était ni plus ni moins que celui qui commettait un acte illicite - un sujet juridique (Foucault, 1976, p.53). Cette conception classique se transforma au $\mathrm{XIX}^{\mathrm{e}}$ siècle avec l'apparition de plusieurs discours - médical et psychiatrique surtout - qui incorporèrent dans le «criminel» une subjectivité. Ainsi, l'auteur de l'acte de sodomie acquiert graduellement une subjectivité, une histoire, une psychologie, une morphologie, une anatomie et une curieuse physiologie qui se cristallisent toutes dans la production d'un nouveau savoir: c'est un homosexuel. Tandis qu'il est maintenant soumis à de puissants discours qui nomment sa condition et qui l'assujettissent, l'homosexuel est cependant en mesure d'y résister grâce à l'acquisition de sa nouvelle identité. Selon Foucault, dès qu'un agent social acquiert une identité d'homosexuel, il peut utiliser cette position et revendiquer sa nouvelle identité de plusieurs façons, soit en s'étalant, en scandalisant, en résistant, soit en acceptant passivement l'idée qu'il est malade. Ainsi, la croissance des perversions, des sexualités non orthodoxes, correspond pour Foucault à un «produit réel de l'interférence d'un type de pouvoir sur les corps et leurs plaisirs » (Foucault, 1976, pp.65-66). C'est dans ce sens que Foucault affirme que le pouvoir n'est ni une institution ni une structure mais «une situation stratégique complexe» (Foucault, 1976, p. 123). Cette conception du pouvoir se distingue d'un pouvoir entendu comme une instance ou une puissance qui étale sa force nocive sur tout le corps social. Chez Foucault, le pouvoir implique plutôt l'idée de relation. d'un rapport de forces entre individus. Ce rapport de forces n'implique pas l'idée d'enfermement ou d'emprisonnement. Il s'agit beaucoup plus d'un mécanisme qui à la fois contraint et incite à l'action; ainsi, l'idée de résistance est au cour du pouvoir:

là où il y a pouvoir, il y a résistance et [...] pourtant, ou plutôt par là même, celle-ci n'est jamais en position d'extériorité par rapport au pouvoir. [...] on est nécessairement «dans» le pouvoir, [...] on ne lui «échappe» pas (Foucault, 1976, pp. 125-126). 
Il est évident que cette conception ne nie en rien les effets de contrôle social crés lors de l'exercice du pouvoir. Mais la production de contrôle (de disciplines ou de normes) qui émane des relations de pouvoir n'est ni unitaire ni unifiante; elle fait partie intégrante d'un rapport de forces qui engendre à la fois des stratégies contraignantes et incitatrices. En d'autres mots, les savoirs produits par les relations de pouvoir n'ont pas d'effets fixes: ils servent à la fois d'instruments de contrôle et d'obstacles, et de points de résistance au contrôle. Foucault illustre ce double mouvement du pouvoir dans le cas du discours fabriquant l'homosexuel:

Or l'apparition au $\mathrm{XIX}^{\mathrm{e}}$ siècle, dans la psychiatrie, la jurisprudence, la littêrature aussi, de toute une série de discours sur les espèces et sous-espèces d'homosexualité, d'inversion, de pédérastie, d' «hermaphrodisme psychique», a permis à coup sûr une très forte avancée des contrôles sociaux dans cette région de «perversité»; mais elle a permis aussi la constitution d'un discours «en retour»: l'homosexualité s'est mise à parler d'elle-même, à revendiquer sa légitimité ou sa «naturalité » et souvent dans le vocabulaire, avec les catégories par lesquelles elle était médicalement disqualifiée. Il n'y a pas d'un côté le discours du pouvoir et, en face, un autre qui s'oppose à lui. Les discours sont des éléments ou des blocs tactiques dans le champ des rapports de forces (Foucault, 1976, p. 134).

\section{c) Pouvoir-savoir et gouvernement}

L'affirmation de Foucault que là où il y a pouvoir il y a nécessairement résistance n'a pas été adéquatement établie dans sa recherche sur la folie, la prison et la sexualité. Alors qu'il soutient que les individus résistent, l'explication qu'il donne de cette résistance est faible. Par exemple, examinons comment il explique la résistance des homosexuels aux discours les assujettissant. Lors d'une entrevue, après la publication de son premier volume sur la sexualité, Foucault dit qu'à un certain moment de notre histoire nous en sommes tous venus à penser l'homosexuel comme manifestant des formes de maladies mentales, physiques ou sexuelles. Et que c'est en:

prenant au pied de la lettre de pareils discours et, par là même, les contournant, [qu']on voit apparaître des réponses en forme de défi: soit, nous sommes ce que vous dites, par nature, maladie ou perversion, comme vous voudrez. Eh bien, si nous le sommes, soyons-le et si vous voulez savoir ce que nous sommes, nous vous le dirons nous-mêmes mieux que vous (Foucault, 1977b, p. 98). 
Ce qui est ambigu, dans l'énoncé de Foucault, c'est comment le sujet, dans ce cas l'homosexuel, est capable d'approprier le discours l'objectivant et de l'utiliser à son avantage. En d'autres mots, quels mécanismes permettent aux sujets d'une population de revendiquer pour eux-mêmes le droit à l'autodétermination?

La question des droits de la personne a été examinée par Foucault d'une façon contournée plutôt qu'explicite. Alors qu'il semble être préoccupé, dans ses écrits, entre 1972 et 1976, par le dévoilement des effets disciplinaires des relations de pouvoir-savoir, il a brièvement posé la question du droit. Dans ses «deux communications» présentées en Italie en $1976^{5}$, il affirme que les relations de pouvoir-savoir sont formellement délimitées par la règle de droit (Foucault, 1980). Mais, il n'a porté son attention au complexe pouvoir-savoir-droit que pour réduire la règle de droit à une idéologie qui dissimulerait ses éléments de domination ${ }^{6}$ (Foucault, 1980, p. 105). Cette conception de la règle de droit renvoie à une vision monarchique du pouvoir, où le pouvoir, le savoir et le droit étaient tous fusionnés dans le corps du souverain. En fait, en 1976, Foucault insiste sur le fait qu'on ne devrait pas évoquer l'ancienne notion de droit pour résister au pouvoir disciplinaire, mais se tourner plutôt vers « la possibilité d'une nouvelle forme de droit. qui devrait être anti-disciplinaire. mais à la fois libérée du principe de souveraineté» (Foucault, 1980, p. 108).

Je pense qu'à la lumière de notre modernité, caractérisée par la séparation du pouvoir, du savoir et du droit du corps du souverain, la recherche d'une nouvelle forme de droit est futile. Lefort a démontré qu'avec l'émergence de la révolution démocratique le corps du roi disparaît en tant qu'ultime fondement du pouvoir, du savoir et du droit, et disparaît du même coup la forme de société associée au monarque (1981, p.64). Ainsi avec la Déclaration des Droits de l'Homme de 1791 s'installe un nouveau point de fixation du droit qui est d'une nature immaîtrisable, «l'homme». Lefort affirme que :

[1]es droits de l'homme ramènent le droit à un fondement qui, en dépit de sa dénomination, est sans figure, se donne comme intérieur à lui et, en ceci, se

5. Le texte de ces communications n'a pas paru en français. Il a été transcrit et traduit par A. Fontana et P. Pasquino dans Michel Foucault, Micro-fisica del Potere (Turin), 1977. La traduction anglaise, "Two Lectures" est dans C. Gordon (ed.), 1980. La traduction française du texte anglais est la mienne.

6. Cette conception des droits qui dissimulent le pouvoir est semblable d'une certaine manière à la conception qu'en a Marx, sauf que pour ce dernier les droits consistent en une représentation camouflant le pouvoir de la propriété privée. Claude Lefort a opposé à celte vision des droits en tant que camouflage une analyse impressionnante de l'indéterminabilité des droits (1981 et 1986). 
dérobe à tout pouvoir qui prétendrait s'en emparer — religieux ou mythique, monarchique ou populaire. Ils sont, en conséquence, en excès sur toute formulation advenue: ce qui signifie encore que leur formulation contient l'exigence de leur reformulation ou que les droits acquis sont nécessairement appelés à soutenir des droits nouveaux. [...] À partir du moment où les droits de l'homme sont posés comme ultime référence, le droit établi est voué au questionnement (Lefort, 1981, pp. 66-67).

Réduire le problème du droit à un processus de dissimulation ${ }^{7} \mathrm{du}$ pouvoir, c'est en quelque sorte confondre l'idéologique avec la valeur symbolique du discours de droit. Le pouvoir, souligne Lefort, peut limiter et même dénier le droit démocratique, «mais [il] est incapable de se priver de sa référence" (1981, p.69). C'est cette dimension symbolique des droits qui nous permet de comprendre le sens des revendications des nouveaux droits, c'est-à-dire de la résistance. Les gens rêsistent aux conditions de leur existence grâce à cette conscience moderne qu'ils ont des droits, grâce à cette tradition des conduites, c'est-à-dire des modes d'existence dans lesquels ils se trouvent. Comme l'affirme Lefort:

Ces droits divers ne s'affirment-ils pas en raison d'une conscience du droit, sans garantie objective, et, tout autant en référence à des principes publiquement reconnus qui se sont pour une part imprimés dans les lois et qu'il s'agit de mobiliser pour détruire les bornes legales auxquelles ils se heurtent (1981, p.71).

En somme, la relation que les individus entretiennent avec l'ordre politique et légal est essentielle pour comprendre le phénomène de la résistance.

La nature de la relation entre l'individu et l'ordre politique est un sujet que Foucault a entrepris dans ses derniers travaux, en particulier ceux sur le «bio-pouvoir » et sur la «bio-politique», ce dernier se référant plus particulièrement à la "gouvernementalité» et au libéralisme en tant qu'art de gouverner (1988, 1989). La notion de «bio-pouvoir» avait été introduite dans son travail sur la sexualité pour désigner les technologies, à la fois globalisantes et individualisantes, par lesquelles les individus sont interpellés en tant que membres d'une population, d'une nation ou d'un État. Cette notion faisait référence à la prolifération de toute une technologie de

7. Alors que Marx, dans La question juive, réduit le droit à des relations bourgeoises ou à la réalité économique, Foucault, dans ses communications de 1976, l'a réduit à une théorie organisée autour du principe de souveraineté. Son travail sur le libéralisme suggère, cependant, la possibilité de penser le droit sans fondement déterminé. 
pouvoir-savoir ayant pour but la prise en charge de la vie par l'investissement du corps, de la santé, des conditions de vie, en fait de «l'espace tout entier de l'existence» (Foucault, 1976, p. 189). La conception du «bio-pouvoir» est significative pour comprendre la résistance car, alors qu' elle représente un mécanisme - globalisant - permettant l'interpellation du sujet en tant que membre d'une population, elle contient en elle-même les éléments pour l'émergence d'un contre-pouvoir ou d'une contre-politique - un mécanisme individualisant. Foucault affirme:

Et contre ce pouvoir encore nouveau au $\mathrm{XIX}^{\mathrm{e}}$ siècle. les forces qui résistent ont pris appui sur cela même qu'il investit - c'est-à-dire sur la vie et l'homme en tant qu'il est vivant. Depuis le siècle passé, [...] ce qui est revendiqué et sert d'objectif, c'est la vie, entendue comme besoins fondamentaux, essence concrète de l'homme, accomplissement de ses virtualités. plénitude du possible. Peu importe s'il s'agit ou non d'utopie; on a là un processus très réel de lutte; la vie comme objet politique a été en quelque sorte prise au mot et retournée contre le système qui entreprenait de la contrôler. C'est la vie beaucoup plus que le droit qui est devenue alors l'enjeu des luttes politiques, même si celles-ci se formulent à travers des affirmations de droit (1976. p. 190-191).

Si la vie, entendue comme essence de l'homme, est revendiquée à travers des affirmations de droit, on ne peut plus continuer de penser le droit comme étant nécessairement relié au souverain. Alors que Foucault n'a pas spécifiquement posé la question des droits de l'homme, ses cours sur la «bio-politique » laissent entendre que le bio-pouvoir - les mécanismes qui ont pour cible la population et dont les objectifs sont de la rendre gouvernable - prend forme dans une rationalité de gouvernement qui est indéterminable, le libéralisme. Dans ce contexte, le droit comme le pouvoir et le savoir sont sans détermination naturelle.

Foucault comprend le libéralisme non pas comme une théorie mais comme un «art de gouverner» qui apparaît vers le XVIII ${ }^{e}$ siècle pour critiquer la raison d'État. Le liberralisme, pour Foucault:

n'est pas un rêve qui se heurte à une réalité et manque à s'y inscrire. Il constitue - et c'est là la raison et de son polymorphisme et de ses récurrences - un instrument critique de la réalité: d'une gouvernementalité antérieure, dont on essaie de se démarquer: d'une gouvernementalité actuelle qu'on tente de réformer et de rationaliser en la révisant à la baisse; d'une gouvernementalité à laquelle on s'oppose et dont on veut limiter les abus (1989, p. 113, c'est moi qui souligne). 
Le libéralisme se distingue de la raison d'État par le fait que son «art de gouverner" ne s'organise pas autour du principe de la souveraineté. Ce qui lui permet de limiter le pouvoir de l'État n'est plus l'idée d'un droit naturel extérieur à l'État, mais une nouvelle rationalité, une gouvernementalité de la vie «se régulant par une réflexion continue» (Foucault, 1989, p. 110). L'idée de réflexivité, ici, est centrale, je pense, car elle fait appel à un mécanisme d'autolimitation inhérent (intrinsèque) au libéralisme. Les gens résistent contre ce qui est, émettent des revendications pour ou contre les formes d'existence dans lesquelles ils vivent au nom de cette rationalité qui a façonné leur vie, qui les a soumis au gouvernement. Ainsi, le libéralisme, entendu comme une rationalité permettant la critique des formes d'existence dans lesquelles les individus se trouvent est, tout comme le droit démocratique dont parle Lefort (1981), immaîtrisable.

Je pense qu'il y a des similitudes entre l'affirmation de Lefort à l'effet que les droits démocratiques sont constitutifs de la politique et de la «nature de l'homme» et l'affirmation de Foucault selon laquelle le liberalisme est une pratique politique de critique de la raison d'État. Premièrement, ils mettent tous les deux l'emphase sur la qualité indéterminable et polymorphe de la rationalité actuelle qui nous constitue en sujets gouvernables, et localisent cette rationalité dans la liberté et le savoir de ceux qui sont gouvernés plutôt que dans le souverain. Deuxièmement, ils soutiennent que le pouvoir, le savoir et le droit caractérisant notre modernité sont sans détermination naturelle. Finalement, ils suggèrent le rôle fondamental de l'affirmation des droits (à l'égalité, à la liberté, à la citoyenneté, etc.) dans la constitution de la politique et des individus.

\section{CONCLUSION}

Nous sommes maintenant bien loin de la critique du savoir conventionnel, en criminologie, sur la réforme du droit. Il serait peut-être utile, en conclusion, de réfléchir sur les possibilités de recherches sur la réforme du droit que l'on peut tirer du travail de Foucault. Je pense qu'un des outils les plus importants de la «bô̂te à outils» que nous offre l'œuvre de Foucault est sa méthodologie. Même si je n'ai pas examiné sa méthodologie de façon explicite, on peut facilement déduire certains principes de l'analyse que j'ai présentée.

Foucault aborde son objet d'étude — la maladie, la prison, la sexualité de façon à comprendre la constitution des sujets et de leur savoirs à travers des stratégies de pouvoir-savoir qui sont spécifiquement historiques et 
culturelles. Cette approche se passe du sujet transcendantal de la phénoménologie, du sujet pensant de l'humanisme libéral, et du sujet vide du structuralisme. Dans un langage plus commun aux sciences sociales, on peut dire que l'approche de Foucault évite de concevoir la réalité sociale en opposition binaire: la structure versus l'agent social. Foucault propose une réalité sociale qui ne se comprend pas comme l'accomplissement d'acteurs sociaux compétents construisant leur monde à travers les pratiques organisées de la vie quotidienne. Elle ne se comprend pas non plus comme une réification d'entités autonomes - les structures - capables d'agir selon une logique indépendante du sujet. À l'objectivisme des structures et au subjectivisme de l'agent social, Foucault oppose une conception relationnelle du monde social.

L'action sociale et la structure s'articulent dans un rapport de pouvoirsavoir. Ces concepts peuvent donc être pensés comme un rapport de forces, une relation, une stratégie dans laquelle sont produits des effets à la fois globalisants - telles les normes qui objectivent l'action - et individualisants - telle la résistance aux normes qui incite l'action. Ainsi, ce qui doit être pris en considération c'est le processus de constitution mutuelle de l'action et de la structure sociale. À l'instar de Foucault, on ne peut plus penser l'action comme l'effet d'une structure (la norme, la discipline, le contrôle). Au primat de la structure, il faut opposer une conception de la réalité sociale dans laquelle l'action et la structure s'articulent dans un processus constitué et constituant qui n' est jamais déterminé, mais toujours contingent. En effet, l'articulation de ce processus, selon Foucault, est spécifiquement historique et culturelle, c'est-à-dire toujours provisoirement et précairement inscrite dans des rapports de forces.

La criminologie a déjà commencé à s' interroger sur l'essentialisme du savoir qu'elle a produit ${ }^{8}$. Cela semble résulter beaucoup plus du défi posé

8 Ce questionnement est plutôt d'ordre épistémologique, voir M.A. Bertrand (1991). Il est intéressant de noter, cependant, que les champions anglophones de la thèse du "nothing works" ont modifie leur approche. Ericson et al. $(1987,1989,1991)$ dans leur recherche sur les médias, examinent la constitution d'un savoir médiatisé sur le crime qui tient compte de la complexité et de la diversité du rapport de forces constituant ce savoir. Cohen (1987), dans sa révvaluation du savoir conventionnel sur les réformes du système de justice, est très explicite quant à l'usage simplifié qu'il a fait - ainsi que bien d'autres - de Foucault:

The ritual quotes from Foucault were pulled out to prove that nothing could escape the awesome power of the state. Legal and disciplinary powers somehow «escape" from the centre, filter out and disperse themselves, and then colonize the furthest extremities ( capillaries") of the social order. $[\ldots]$ But this was surely a one-sided reading of a theory whose main thrust 
par ce qu'on nomme le postmodernisme, que par «l'effet Foucault». Mais en fait, Foucault, était-il moderne ou postmoderne? Cette question qui hante plusieurs - et nous-mêmes dans ce numéro spécial sur Foucault et sur le postmodernisme - a été débattue à maintes reprises par plusieurs philosophes, critiques littéraires et autres (Arac, 1988). Quoi qu'on en décide, pour nous en criminologie, je pense qu'il vaudrait mieux nous attarder à le (re)lire.

\section{BIBLIOGRAPHIE}

ARAC, Jonathan (ed). (1988), After Foucault: Humanistic Knowledge, Postmodern Challenges. New Brunswick, Rutgers University Press.

BEN-YEHUDA, Nachman (1985), Deviance and Moral Boundaries. Chicago, University of Chicago Press.

(1986), "The Sociology of Moral Panics: Towards a New Synthesis", The Sociological Quarterly, vol. 24, $\mathrm{n}^{\circ} 4$, p. 495-514.

BARRET-KRIEGEL, B. (1989), «Michel Foucault et l'État de police», in Michel Foucault philosophe: rencontre internationale Paris 9, 10, 11 janvier 1988. Paris, Éditions du Seuil.

BERTON, A. (1981), «Un entretien avec Michel Foucault», (vidécassette), Université catholique de Louvain.

BERTRAND, M.A. (1991), "On the importance of epistemology for feminist criticism and on the potentialities of various epistemologies", in Bertrand, M.A., K. Daly, and D. Klein (ed.), Proceedings of the International Feminist Conference on Women, Law, and Social Control, Mont Gabriel, Québec, 18-21 juillet.

CHAN, J. and ERICSON, R.V. (1991), Decarceration and the Economy of Penal Reform, Research Monograph $\mathrm{n}^{\circ} 14$, Toronto, Centre of Criminology, University of Toronto.

COHEN, S. (1979), "The Punitive City: Notes on the Dispersal of Social Control", Contemporary Crisis, 3, p. 340-355.

was to deny the privileged position given to the central state apparatus. $I \ldots . \mid$ To wheel in Foucault to support a simple theory of a concentrated and centralized state power which infiltrates all other local forms of social control is hardly justified (pp. 377-378).

Cohen propose de poursuivre les recherches sur la réforme en mettant l'emphase sur les succès plutôt que les échecs. Pour ce faire, il faut évidemment reconceptualiser la notion de pouvoir. Je remercie Maeve W. McMahon de m'avoir signalé cet article de Cohen. 
COHEN, S. (1983), "Social-Control Talk: Telling Stories About Correctional Change". in D. Garland et P. Young (ed.), The Power to Punish: Penality and Social Analysis. London, Heinemann Educational Books.

COHEN, S. (1985), Visions of Social Control: Crime, Punishment and Classification. Cambridge, Polity Press.

COHEN, S. (1987), "Taking decentralization seriously: values, visions and policies", in Transcarceration: essays in the sociology of social control. J. Lowman, R.J. Menzies, T.S. Palys (ed.), Hants, Gower.

DELEUZE, G. (1975), «Écrivain non: un nouveau cartographe», in Critique, 31, 343, pp. 1207-1227.

ERICSON, R.V. (1987), "The State and Criminal Justice Reform", in R.S. Ratner and J.L. McMullan (ed.), State Control : Criminal Justice Politics in Canada. Vancouver, University of British Columbia Press.

ERICSON, R.V., and BARANEK, P.M. (1982), The Ordering of Justice: a Study of Accused Persons as Dependants in the Criminal Process. Toronto, University of Toronto Press.

ERICSON. R.V., BARANEK, P. and CHAN, J. (1987), Visualizing Deviance. Toronto, University of Toronto Press and Milton Keynes, Open University Press.

(1989), Negotiating Control. Toronto, University of Toronto Press and Milton Keynes, Open University Press.

(1991), Representing Order. Toronto, University of Toronto Press and Milton Keynes, Open University Press.

EWALD, F. (1975), «Anatomie et corps politiques $»$, in Critique, 31, 343, p. 12281265.

FOUCAULT M. (1975), Surveiller et punir: naissance de la prison. Paris, Gallimard.

(1976), Histoire de la sexualité : la volonté de savoir. Paris, NRF, Gallimard.

(1977a), «L'œil au pouvoir », entretien recueilli par J.P. Barou et M. Pecrot, in J. Bentham, Le Panoptique. Paris, Belfond, p. 8-31.

(1977b), «Non au sexe roi», propos recueillis par Bernard-Henri Lévy, in Le Nouvel Observateur, $\mathrm{n}^{\circ}$ 644, 12 mars, p. 92- 130 .

(1980), “Two Lectures", in Gordon, Colin (ed.), Power/Knowledge: Selected Interviews and Other Writings 1972-1977. Sussex, The Harvester Press, p.98108.

(1986), «La gouvernementalité», in Actes, n 54, pp. 6-15.

(1988), "An aesthetics of existence", in L.D. Kritzman (ed.), Michel Foucault: Politics, Philosophy, Culture, Interviews and Other Writings, 1977. 1984. New York, Routledge, Chapman \& Hall, p. 47-53.

(1989) Résumé de cours 1970-1982, (Conférences, essais et leçons du Collège de France). Paris, Julliard. 
(1991) "Governmentality", in G. Burtchell, C. Gordon, et P. Miller (ed.), The Foucault Effect: Studies in Governmentality. Hertfordshire, Harverster Wheatsheaf, p. 87-104.

GARLAND, D. (1985), Punishment and Welfare: a History of Penal Strategies. Aldershot, Gower, 1985.

(1986), "Foucault's Discipline and Punish, An Exposition and Critique", American Bar Foundation Research Journal, pp. 847-881.

(1990), Punishment and Modern Society: A Study in Social Theory. Chicago, The University of Chicago Press.

GIFFEN, James P. et Sylvia LAMBERT (1988), "What Happened on the Way to Law Reform?" in Judith C. Blackwell et Patricia G. Erickson (ed.), Illicit Drugs in Canada: A Risky Business. Toronto, Nebon, p. 345-369.

GORDON, Colin (ed.) (1980), Power/Knowledge: Selected Interviews and Other Writings 1972-1977. Sussex, The Harvester Press.

GUSFIELD, J. (1981), The Culture of Public Problems: Drinking Driving and the Symbolic Order. Chicago, University of Chicago Press.

HALL, S., et al. (1978), Policing the Crisis: Mugging, the State and Law and Order, London, The Macmillan Press.

IGNATIEFF, M. (1978), A Just Measure of Pain: the Penitentiary in the Industrial Revolution. New York, Pantheon Books.

IGNATIEFF, M. (1983), "State, Civil Society and Total Institutions: a Critique of Recent Social Histories of Punishment", in S. Cohen et A.T. Scull (ed.), Social Control and the State. Oxford, Martin Robertson.

LACOMBE, D. (a venir) The Powers of Pornography: a Study of Resistance, Social Science and Law Reform. Toronto, University of Toronto Press.

LEFORT, C. (1981), L'invention démocratique: les limites de la domination totalitaire. Paris, Fayard.

LEFORT, C. (1986), Essais sur le politique: $X I X^{e}-X X^{e}$ siècles. Paris, Éditions du Seuil.

MCBARNETT, D. (1981), Conviction: Law, the State and the Construction of Justice. London, MacMillan.

MCMAHON, Maeve W. (1992), The Persistent Prison? Rethinking Decarceration and Penal Reform. Toronto, University of Toronto Press.

ROTHMAN, D. (1971), The Discovery of the Asylum: Social Order and Disorder in the New Republic. Toronto, Little Brown.

ROTHMAN, D. (1980), Conscience and Convenience: the Asylum and its Alternatives in Progressive America. Boston, Little Brown.

SHEARING, C.D., et P.S. STENNING (1984), "From the Panopticon to Disney World: The Development of Discipline", in A.N. Doob et E.L. Greenspan (ed.), Perspectives in Criminal Law. Aurora, Ontario, Canada Law Book. 
SMALL, N. (1988), "AIDS and Social Policy”, Critical Social Policy, 21, p.9-29

SMART, C. (1989), Feminism and the Power of Law. London, Routledge.

WATNEY, S. (1987), Policing Desire: Pornography, AIDS and the Media. London, Methuen. 